coronary flow and is an important predictor of coronary microvascular function. A variety of environmental stimuli have been shown to affect CFR but little is known about the genetic component of CFR. To characterise the genetics of CFR we initially measured in vivo blood pressure (BP) and ex vivo cardiac phenotypes including CFR in two inbred rat strains, Brown Norway $(\mathrm{BN})$ and Spontaneously Hypertensive Rat (SHR) which is a genetic model for hypertension and microvascular dysfunction. We then studied $\mathrm{BP}$ and coronary flow (CF) phenotypes in $\mathrm{F}_{1}$ and $\mathrm{F}_{2}$ crosses derived from $\mathrm{BN}$ and SHR to estimate the heritability of CFR and its relationship with BP.

Methods Animals were anaesthetized using a mixture of Oxygen and Isoflurane. BP was measured invasively by cannulation of carotid artery. Following BP measurement hearts were excised and rapidly transferred to the ex vivo perfusion apparatus where retrograde perfusion was established using the Langendorff technique. Hearts were perfused with Carbogen buffered Kreb's solution and paced constantly at $360 \mathrm{bpm}$. A fluid filled balloon was placed in the left ventricular (LV) cavity to measure the pressure indices. CF, LV developed pressure, myocardial contractility ( $\mathrm{LV} \mathrm{dP} / \mathrm{dt}_{\max }$ ) and myocardial relaxation ( $\left.\mathrm{LV} \mathrm{dP} / \mathrm{dt}_{\mathrm{min}}\right)$ were recorded at baseline, during peak hyperaemia, regional ischaemia (induced by ligation of the proximal left anterior descending artery) and reperfusion.

Results 1) CFR differs significantly between the two inbred parental rat strains. $\left(\mathrm{BN}=2.1 \pm 0.32, \mathrm{SHR}=1.5 \pm 0.18, \mathrm{p}=2.6 \times 10^{-7}, \mathrm{n}=16\right.$ each). 2) Heritability of CFR: Broad sense heritability (the proportion of total phenotypic variance attributable to total genetic variance) for CFR is $62 \%$ indicating a large and previously unrecognised genetic component of CFR. 3) Relationship between CFR and BP: We did not find statistically significant correlation between CFR and BP in the $\mathrm{F}_{2}$ intercross $(\mathrm{r}=0.11, \mathrm{p}=0.11, \mathrm{n}=176)$. 4) Relationship between CF and myocardial relaxation ( $\left.\mathrm{LV} \mathrm{dP} / \mathrm{dt}_{\mathrm{min}}\right)$ : $\mathrm{LV}$ $\mathrm{dP} / \mathrm{dt}_{\text {min }}$ correlated strongly with CF during all stages of the experiment (baseline $\mathrm{CF}, \mathrm{r}=-0.36, \mathrm{p}<0.0001$, reperfusion $\mathrm{CF}$, $\mathrm{r}=-0.40, \mathrm{p}<0.0001$ ).

Conclusions Our results demonstrate that CFR has a significant genetic component and is largely independent of BP effects. Furthermore we demonstrate a very significant relationship between $\mathrm{CF}$ and $\mathrm{LV} \mathrm{dP} / \mathrm{dt}_{\min }$ indicating a link between LV diastolic dysfunction and impaired CF. Using 768 SNP genotyping assay for linkage mapping and gene expression analysis with Affymetrix rat gene chip, we will determine the quantitative trait loci and transcripts associated with CFR to improve our understanding of the genomic architecture of CFR.

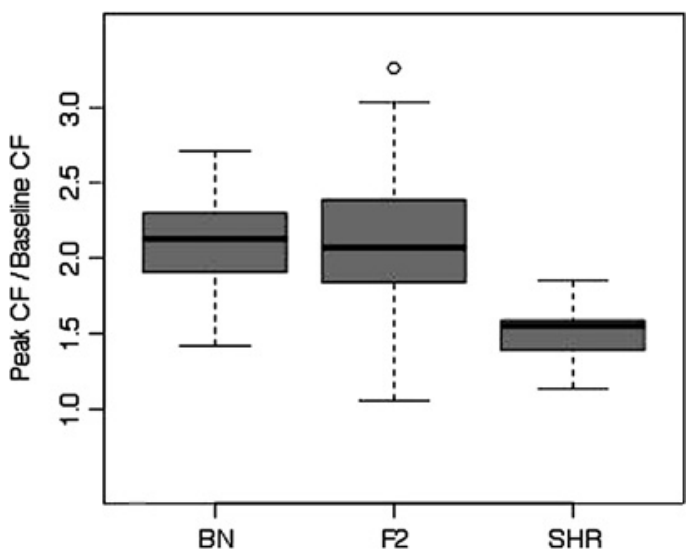

Abstract 73 Figure 1 Coronary flow reserve.

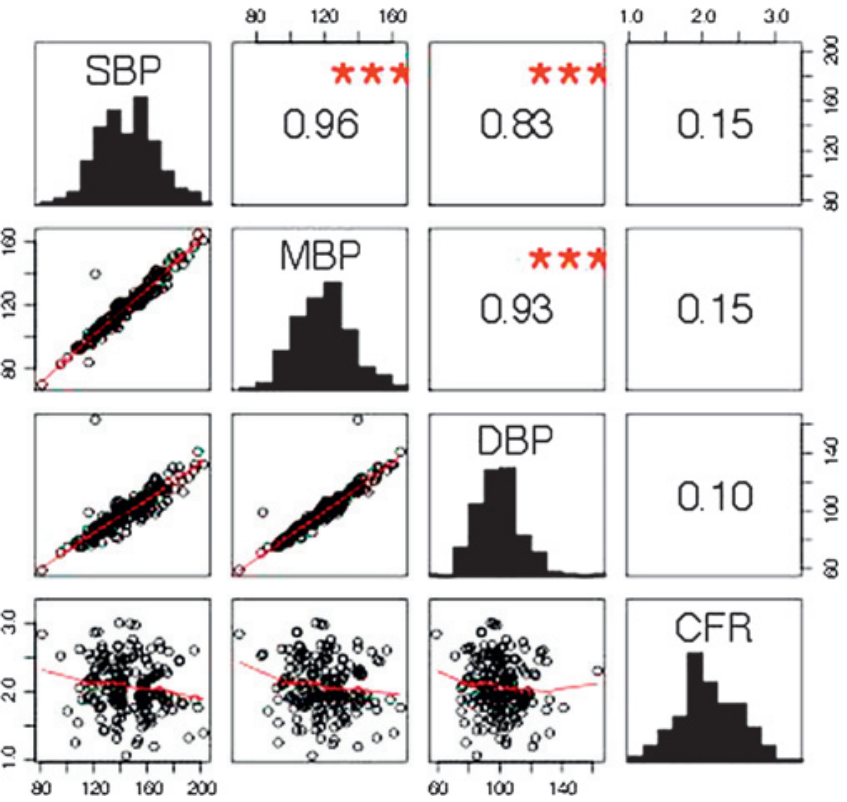

Abstract 73 Figure 2 Correlation between BP and CFR.

\section{MECHANISTIC STUDY FOR THE ROLE OF ADVANCED GLYCATION END PRODUCTS IN THE DEVELOPMENT OF DIABETIC HEART FAILURE}

doi:10.1136/heartjnl-2011-300198.74

Z Hegab, T M A Mohammed, L Neyses, M Mamas. Manchester University, Manchester, UK

Advanced glycation end products (AGEs) are thought to play a crucial role in the development of diabetic complications including heart failure, a leading cause of morbidity and mortality in diabetic patients. However, the molecular mechanisms that underlie the pathophysiological contribution of AGEs to heart failure development are not yet fully understood. We therefore investigated the effects and mechanisms of action of AGEs on isolated neonatal rat cardiomyocytes (NRCM). Standard molecular techniques were applied. Western blot showed that RAGE receptor is expressed in NRCM and adult mouse cardiomyocytes. Incubation of NRCM for $24 \mathrm{~h}$ with AGEs showed a dose dependant reduction of calcium transient amplitude with a maximum of $52 \%$ at $1 \mathrm{~g} / \mathrm{l} \quad(\mathrm{p}<0.01)$ accompanied with $32 \%$ reduction in SR calcium content with no significant changes in the protein expression of calcium handling proteins. We demonstrated a $24 \%$ increase $(p<0.01)$ in the production of reactive oxygen species ROS in AGE treated cardiomyocytes mediated through increased NADPH oxidase activity $(p<0.05)$. Subsequent translocation of NF-KB, a transcriptional factor from the cytoplasm to the nucleus together with increased NF-KB activity resulted in a $56 \%$ increase in iNOS gene protein expression $(p<0.01)$, a downstream target of NF-KB. The latter was associated with $10 \%$ increase in NO production $(p<0.05)$ with subsequent nitrosylation of the Ryanodine receptor shown through immunofluoresence. Changes in calcium transient were completely inhibited when we incubated the cardiomyocytes with inhibitors of NADPH oxidase, NOS or NF-KB prior to their incubation with AGEs. In conclusion, AGEs directly decline cardiomyocytes function through binding to their RAGE receptor leading to calcium handling impairment through increased ROS production inducing activation and translocation of NF-KB to the nucleus. The latter increased transcription of iNOS with increased NO production. Coexistence 
of ROS and NO favours the production of peroxynitrite that is capable of nitrosylation of key cellular proteins such as the Ryanodine receptor that has a crucial role in cardiac excitationcontraction coupling. This study provides novel insights into the mechanisms of cardiac damage in diabetes that occur independent of vascular disease through AGEs.

\section{OPTIMISATION OF MEDICAL THERAPY AFTER CARDIAC RESYNCHRONISATION: A NURSING OPPORTUNITY NOT TO BE MISSED}

doi:10.1136/heartjnl-2011-300198.75

${ }^{1} \mathrm{~S}$ J Russell, ${ }^{2} \mathrm{~J}$ Bell, ${ }^{3} \mathrm{~L}$ Edmunds, ${ }^{4} \mathrm{~J}$ Davies, ${ }^{3} \mathrm{H}$ Rose, ${ }^{3} \mathrm{Z} \mathrm{R}$ Yousef. ${ }^{1}$ Wales Heart Research Institute, Cardiff, UK; ${ }^{2}$ Cardiff University, Cardiff, UK; ${ }^{3}$ University Hospital of Wales, Cardiff, UK; ${ }^{4}$ University Hospital Llandough, Cardiff, UK

Introduction Cardiac resynchronisation therapy (CRT) is indicated in patients with left ventricular dysfunction ( $E F \leq 35 \%)$, electromechanical dyssynchrony, and limiting heart failure (HF) symptoms despite optimal medical therapy. In many cases target doses of HF medications prior to CRT are not achieved due to bradycardia and/or limiting hypotension. CRT however provides bradycardia backup and improved haemodynamics, thus providing an opportunity to further optimise HF medical therapies known to confer substantial morbidity and mortality benefits. We conducted the present study to evaluate the potential to further optimise medical treatments in patients receiving CRT within the framework of nurse-led pre and post CRT clinics.

Methods Our unit operates an integrated CRT service with preassessment, implantation, and follow-up components. Pre-assessment and follow-up incorporate dedicated HF nurse clinics to support protocol-driven optimisation of medical therapies. We therefore conducted a retrospective analysis of our CRT database over a 9-month period to quantify the frequency of use, and dose of HF medications ( $\beta$ blockers; $\beta B$, angiotensin converting enzyme inhibitors: ACE-I or angiotensin receptor blockers: ARB, aldosterone antagonists, digoxin, and loop diuretics) before and 6 months after CRT. Total daily dose equivalences within each class of medication (bisoprolol for $\beta B$, lisinopril for ACE-I/ARB, spironolactone for aldosterone antagonists, and frusemide for loop diuretics) and titration protocols were based on National Institute of Clinical Excellence guidelines for HF (guideline 5).

Results Between October 2009 and Jun 2010, 74 patients (age: $67 \pm 11 \mathrm{yrs}, 86 \%$ male) underwent implantation of a CRT device. All

Abstract 75 Table 1 Heart Failure nurse supervised use of medications before and 6 months After CRT

\begin{tabular}{|c|c|c|c|}
\hline & Pre-CRT & Post-CRT & p Value \\
\hline \multicolumn{4}{|c|}{$\beta$-Blocker: exemplar bisoprolol } \\
\hline Frequency of use & $78 \%$ & $88 \%$ & \multirow[t]{2}{*}{$<0.01$} \\
\hline Daily dose equivalent & $5.3 \pm 3.1 \mathrm{mg} /$ day & $6.9 \pm 3.2 \mathrm{mg} / \mathrm{day}$ & \\
\hline \multicolumn{4}{|c|}{ ACE-I/ARB: exemplar lisinopril } \\
\hline Frequency of use & $93 \%$ & $93 \%$ & \multirow[t]{2}{*}{0.02} \\
\hline Daily dose equivalent & $11.8 \pm 4.5 \mathrm{mg} / \mathrm{day}$ & $12.4 \pm 3.8 \mathrm{mg} / \mathrm{day}$ & \\
\hline \multicolumn{4}{|c|}{ Aldosterone Antagonist: exemplar spironolactone } \\
\hline Frequency of use & $32 \%$ & $28 \%$ & \multirow[t]{2}{*}{0.32} \\
\hline Daily dose equivalent & $16.6 \pm 6.2 \mathrm{mg} / \mathrm{day}$ & $15.5 \pm 7.3 \mathrm{mg} / \mathrm{day}$ & \\
\hline \multicolumn{4}{|c|}{ Cardiac Glycoside: exemplar digoxin } \\
\hline Frequency of use & $43 \%$ & $48 \%$ & \multirow[t]{2}{*}{$<0.01$} \\
\hline Daily dose equivalent & $83.8 \pm 11.4 \mu \mathrm{g} / \mathrm{day}$ & $96.1 \pm 13.6 \mu \mathrm{g} / \mathrm{day}$ & \\
\hline \multicolumn{4}{|c|}{ Loop Diuretic: exemplar frusemide } \\
\hline Frequency of use & $100 \%$ & $100 \%$ & \multirow[t]{2}{*}{$<0.01$} \\
\hline Daily dose equivalent & $63.0 \pm 5.8 \mathrm{mg} / \mathrm{day}$ & $48.1 \pm 8.4 \mathrm{mg} / \mathrm{day}$ & \\
\hline
\end{tabular}

patients attended the pre and post CRT nurse clinic to optimise medical therapies and provide adjunctive HF support. Abstract 75 table 1 describes the frequency of use and daily dose equivalent of each class of medication used in the patients prior to and 6 months after device implantation. The frequency of $\beta \mathrm{B}$ and digoxin use increased by $10 \%$ and $5 \%$ respectively. In addition, the dose of $\beta B$, ACE-I/ARB, and digoxin significantly increased, while the dose of loop diuretics significantly reduced in the 6 months after CRT implantation.

Conclusions The beneficial haemodynamic and pacing profiles provided by CRT offer important opportunities to further optimise heart failure medications after device implantation. In a dedicated nurse-led CRT follow-up clinic, we successfully initiated $\beta$ blockers and digoxin in previously naive patients, and significantly uptitrated the doses of $\beta$ blockers, ACE-I/ARB, and digoxin, while significantly reducing loop diuretic use in the 6 months after device implantation.

\section{EXPANDING THE ROLE OF CARDIAC CARE UNIT NURSES TO REDUCE TIME TO TREATMENT FOR PATIENTS REQUIRING PRIMARY ANGIOPLASTY}

doi:10.1136/heartjnl-2011-300198.76

S Young, G Pretsell, A Gibbins, G Dixon, A de Belder. Royal Sussex County Hospital, Brighton, UK

Introduction In Brighton, UK, 24-h Primary Angioplasty has been used for the treatment of ST segment elevation myocardial infarction (STEMI) since October 2008, with local patients being admitted via the Accident and Emergency (A\&E) department. With the publication of the National Infarct Angioplasty project report (DH 2008) it was evident that direct admission into the cardiac catheter lab from the ambulance could further reduce time to treatment. Call to Balloon time (CTBT) $<150$ mins is a nationally recognised indicator measuring the time the patient first calls for professional help (usually the ambulance) to the opening of the coronary artery on the catheter lab Abstract 76 table 1.

\section{Abstract 76 Table 1}

\begin{tabular}{lll}
\hline & $\%$ CTBT $\leq 150$ mins & Median CTBT \\
\hline Financial Year 2009-2010 & 59/78 76\%* & 125 mins \\
Quarter 1 (April-June 2010) & $32 / 3689 \%$ & 111 mins \\
Quarter 2 (July-September 2010) & $44 / 4598 \% *$ & 99 mins \\
\hline
\end{tabular}

${ }^{*} \mathrm{p}=0.0013$.

Methods The on-call cardiology team are non-resident out of hours It was therefore agreed the point of contact and immediate decision making would lie with the Cardiac Care Unit (CCU) nurses. A pathway was developed following consultation with the multidisciplinary team at an educational and mapping day, and risks were addressed. It was agreed that the ambulance crew would telephone the CCU nurse who would review the clinical history and the telemetry ECG. They would then make the decision to activate the catheter lab team. Patient Group Directions for the administration of GTN, diamorphine, metoclopramide and clopidogrel were developed so that immediate treatment could be delivered by the CCU nurse without medical prescription before the cardiac catheter lab team arrived, if required. The nurses were trained in their use and assessed as competent. Nurses were already competent in ECG interpretation, defibrillation, cannulation and venepuncture. Nursing documentation was developed to prioritise the patient's emergency care. CTBT were monitored. 